\title{
Efeitos de Conseqüências Programadas sobre o Comportamento de Seguir Regras
}

\author{
Carla Cristina Paiva Paracampo \\ Luiz Carlos de Albuquerque \\ Andréa Fonseca Farias \\ Bruna Nogueira Carvalló \\ Ana Rachel Pinto \\ Universidade Federal do Pará
}

\begin{abstract}
RESUMO
Investigando variáveis responsáveis pela manutenção do seguir regras, 16 crianças foram expostas a um procedimento de escolha segundo o modelo. A tarefa era tocar um dos estímulos de comparação na presença de uma luz. O comportamento era estabelecido por regra na Fase 1. As contingências na Fase 1 eram revertidas na Fase 2, restabelecidas na Fase 3 e novamente revertidas na Fase 4. As condições diferiram quanto à natureza e ao tipo de conseqüências programadas. O seguimento da regra tendeu a ser abandonado quando passou a produzir um som ou "Errado" e tendeu a ser mantido quando deixou de produzir bombons ou "Certo". Os resultados ampliam a generalidade da sugestão de que o seguimento de regras tem maior probabilidade de ser abandonado quando produz conseqüências aversivas do que quando produz outras conseqüências e sugerem que a manutenção desse comportamento depende, em parte, do tipo de conseqüência por ele contatada.

Palavras-chave: controle por regras; tipo de conseqüências; natureza das conseqüências.
\end{abstract}

\section{ABSTRACT \\ The Effects of Programmed Consequences on Rule-Following}

In an investigation of variables responsible for rule-following, 16 children were exposed to a matching-to-sample procedure. The task involved touching the comparison stimuli when a light came on. In Phase 1, the behavior was established by a rule. In Phase 2, the contingencies in Phase 1 were reversed, re-established in Phase 3 and repeated again in Phase 4. The conditions differed with regard to the nature of the programmed consequences. Rule-following tended to cease in the presence of a tone or the word "Wrong", and was maintained when candy or praise ("Correct") ceased. These results extend the suggested generality that the probability of ceasing rule-following increases when it produces aversive consequences rather than others, and indicates that the maintenance of the behavior depends, in part, on the type of consequence encountered.

Keywords: rule-following; type of consequences; nature of consequences.

Grande parte do repertório humano é estabelecido por regras ${ }^{2}$, sendo raros os exemplos de comportamento humano puramente controlado por contingências. Considerando isto, os estudos sobre o comportamento controlado por regras têm procurado, principalmente, esclarecer o papel do ambiente verbal, público e privado, no controle do comportamento humano (L. C. Albuquerque, 2001, 2005; Andronis, 1991; Baron \& Galizio, 1983; Braga, Albuquerque \& Paracampo, 2005; Catania, 1998; Catania, Matthews
\& Shimoff, 1990; Catania, Shimoff, \& Matthews, 1989; Cerutti, 1989; Chase \& Danforth, 1991; S. C. Hayes, 1986; S. C. Hayes \& Hayes, 1989; Lowe, 1979; Malott, 1989; Schlinger, 1993; Schlinger \& Blakely, 1987; Schlinger \& Blakely, 1994; Simonassi, Tourinho \& Silva, 2001; Skinner, 1963, 1969, 1974, 1989; Zettle \& Hayes, 1982), identificar as variáveis responsáveis pela formação do repertório de seguir regras (L. C. Albuquerque, 2005; Baron \& Galizio, 1983; Catania, 1998; Cerutti, 1989; S. C. Hayes \& Hayes, 1989; L. J. Hayes, Thompson \& Hayes, 1989; 
O’Hora, Barnes-Holmes, Roche \& Smeets, 2004; Okoughi, 1999; Robles \& Gil, 2006; Schmidt \& de Souza, 2002; Skinner, 1969, 1989; Streifel, Wetherby \& Karlan, 1976) e as variáveis responsáveis pela manutenção desse comportamento (L. C. Albuquerque, de Souza, Matos \& Paracampo, 2003; Barret, Deitz, Gaydos \& Quinn, 1987; Cerutti, 1991; DeGrandpre \& Buskist, 1991; Galizio, 1979; S. C. Hayes, Brownstein, Zettle, Rosenfarb \& Korn, 1986; S. C. Hayes, Zettle \& Rosenfarb,1989; Joyce \& Chase, 1990; LeFrancois, Chase \& Joyce, 1988; Martinez \& Tomayo, 2005; Newman, Buffington \& Hemmes, 1995; Otto, Torgrud \& Holborn, 1999; Paracampo \& Albuquerque, 2004; Paracampo, de Souza, Matos \& Albuquerque, 2001; Shimoff, Catania \& Matthews, 1981; Torgrud \& Holborn, 1990; Weiner, 1970).

Entre os estudos que têm investigado as variáveis responsáveis pela manutenção do comportamento controlado por regras, alguns têm manipulado variáveis históricas que antecedem o seguimento de regras (L. C. Albuquerque \& cols., 2003; L. C. Albuquerque, Matos, de Souza \& Paracampo, 2004; L. C. Albuquerque, Reis \& Paracampo, 2006; L. C. Albuquerque \& Silva, 2006; DeGrandpre \& Buskist, 1991; Dixon \& Hayes, 1998; LeFrancois \& cols., 1988; Martinez \& Tomayo, 2005; Monteles, Paracampo \& Albuquerque, 2006; Okoughi, 1999; V. L. Oliveira \& Albuquerque, 2007; C. I. Oliveira, Castro, Simonassi \& Vieira, 2002; Silva \& Albuquerque, 2007), e outros têm manipulado conseqüências programadas para este comportamento (N. M. A. Albuquerque, Paracampo \& Albuquerque, 2004; Baron, Kaufman \& Stauber, 1969; Galizio, 1979; S. C. Hayes \& cols., 1986; Shimoff \& cols., 1981; Otto \& cols., 1999; Paracampo \& Albuquerque, 2004; Paracampo \& cols., 2001; Weiner, 1970). O presente estudo se insere nessa última linha de investigação.

Os estudos que têm investigado o papel de conseqüências programadas na manutenção do seguimento de regras, em geral, têm exposto os participantes (geralmente estudantes universitários ou crianças) a uma instrução correspondente às contingências programadas e depois, sem sinalização, as contingências são alteradas. Observa-se se o comportamento previamente estabelecido pela instrução muda ou não acompanhando a mudança nas contingências. Em geral, tem sido observado, após as mudanças nas contingências, que o seguimento de regras tende a ser mantido quando ele deixa de ser reforçado (Paracampo \& cols., 2001; Santos, Paracampo \& Albuquerque, 2004;
Weiner, 1970) ou quando não produz tanto reforços quanto poderia produzir caso mudasse acompanhando as mudanças nas contingências (S. C. Hayes \& cols., 1986; Shimoff \& cols., 1981; Otto \& cols., 1999) e tende a ser abandonado quando ele produz perda de reforçadores (N. M. A. Albuquerque \& cols., 2004; Galizio, 1979; Paracampo \& Albuquerque, 2004).

Resultados como estes, têm levado alguns autores a sugerir que o seguimento de regras seria mais provável de ser abandonado quando produz conseqüências aversivas do que quando produz outros tipos de conseqüências (Baron \& Galizio, 1983; Chase \& Danforth, 1991; Galizio, 1979; LeFrancois \& cols., 1988; Paracampo \& Albuquerque, 2004; Perone, Galizio \& Baron, 1988). Contudo, esta sugestão está apoiada em resultados obtidos em estudos diferentes (citados no final do parágrafo anterior) e, deste modo, não fica claro se aquelas diferenças de resultados ocorreram devido às diferenças nos tipos de conseqüências contatadas pelo comportamento de seguir regras ou devido a algum outro fator. Além disso, como a maior parte dos estudos, nessa linha de investigação, tem avaliado os efeitos das conseqüências produzidas pelo comportamento de seguir regra, utilizando como conseqüências pontos trocados por dinheiro ou fichas trocadas por brinquedos, a generalidade dessa sugestão está restrita a estas conseqüências. Assim, não está claro se o seguimento de regras também seria abandonado, caso produzisse outras conseqüências, além de perda de pontos ou fichas. Do mesmo modo, também não está claro se este comportamento seria mantido, após a mudança nas contingências, caso deixasse de produzir outras conseqüências reforçadoras, além de pontos ou fichas. Ou seja, não está claro se conseqüências de naturezas diferentes, apresentadas contingentes a manutenção ou não do comportamento de seguir regra, exerceriam os mesmos efeitos daquelas tradicionalmente utilizadas.

O presente estudo procurou avaliar se conseqüências sociais e não-sociais, como a obtenção de bombons ou a apresentação da verbalização "Certo" e a apresentação de um estímulo sonoro ou apresentação da palavra "Errado", têm funções similares às de ganho, não obtenção ou perda de pontos ou fichas no controle do comportamento governado por regras. Também procurou avaliar, tanto entre participantes quanto em um mesmo participante, se a manutenção do seguimento de regras depende do tipo de conseqüência por ele contatada. Para tanto, foi utilizado um 
procedimento de escolha de acordo com o modelo, adaptado do desenvolvido por Paracampo (1991).

\section{MÉTODO}

\section{Participantes}

Participaram do estudo 16 crianças com idades variando entre 7 e 9 anos, sem história experimental prévia, cursando a $1^{\text {a }}$ ou $2^{\text {a }}$ série do Ensino Fundamental. Todas as crianças foram previamente autorizadas pelos responsáveis a participar da pesquisa por intermédio de um formulário de consentimento assinado pelos mesmos. Só participaram do experimento as crianças que após a autorização dos responsáveis concordaram em participar.

\section{Material}

Foi utilizada uma mesa retangular de madeira pintada de branco, cujo tampo tinha uma abertura coberta por uma placa de acrílico leitoso, sob a qual ficavam quatro lâmpadas fluorescentes de 20 watts, duas de cor vermelha e duas de cor amarela. Separando o experimentador do participante, sobre a mesa, havia um anteparo de madeira subdividido em três compartimentos iguais, dentro dos quais ficavam os arranjos de estímulos previamente preparados. Em frente ao terceiro compartimento, à direita do experimentador, ficavam três interruptores, dois para o controle das lâmpadas fluorescentes e um para controle de som.

Foram utilizados como estímulos discriminativos e condicionais 30 diferentes arranjos de estímulos. Cada arranjo de estímulos continha três cartões com desenhos (uma bola, uma lua etc); dois desenhos eram sempre iguais entre si e o terceiro era diferente. Um cartão contendo um dos desenhos iguais era colado no topo da folha (estímulo modelo) e os outros dois mais abaixo e lado a lado (estímulos de comparação). A combinação dos estímulos era aleatória, assim como a ordem de apresentação dos 30 arranjos. Como estímulos contextuais foram utilizadas lâmpadas coloridas acesas. O desempenho dos participantes era registrado por um observador (que ficava sentado atrás do participante) em um protocolo de registro previamente preparado, e era também gravado em vídeo, para análises posteriores.

\section{Procedimentos}

Após ser convidada a participar de um jogo, a criança era conduzida à sala experimental onde participante e experimentador ficavam sentados à mesa experimental, frente a frente. O experimentador apresentava oralmente ao participante uma instrução e, logo após, passava a apresentar os arranjos de estímulos. Em cada tentativa, um arranjo de estímulos era apresentado ao participante e, em seguida, uma das lâmpadas era acesa. Na presença desses estímulos o participante deveria tocar com o dedo apenas um dos estímulos de comparação. Caso a resposta estivesse de acordo com as contingências de reforço programadas (resposta correta), dependendo da condição e da fase experimental, era colocado no recipiente próximo ao participante um bombom ou era dito “Certo”, a lâmpada era apagada, o arranjo retirado, um novo arranjo era apresentado e outra lâmpada era acesa, iniciando uma nova tentativa. Caso a resposta fosse considerada incorreta, dependendo da condição e da fase experimental, ocorria o seguinte: a) um som era apresentado, a lâmpada era apagada e o arranjo de estímulos retirado; b) era dito "Errado", a lâmpada era apagada e o arranjo de estímulos retirado; ou, c) a lâmpada era apagada e o arranjo de estímulos retirado, sem que o som ou o estímulo verbal "Errado" fossem apresentados, isto é, nestes casos a resposta incorreta não era seguida por uma conseqüência específica apresentada pelo experimentador.

Os participantes foram distribuídos em quatro condições experimentais. Cada condição foi realizada com quatro participantes e era constituída de quatro fases. Nas quatro condições, a Fase 1 era iniciada com a apresentação de uma instrução correspondente às contingências de reforço programadas para a fase e o início de cada uma das Fases 2, 3 e 4 era marcado apenas pela mudança, não sinalizada, nas contingências programadas. As quatro condições diferiam entre si quanto à natureza e ao tipo de conseqüência programada para as respostas nas Fases 2 e 4, como indicado na Tabela 1. As Condições 1 e 2 também diferiam das Condições 3 e 4 quanto à conseqüência descrita na instrução correspondente. 
TABELA 1

Esquematização do Procedimento

\begin{tabular}{|c|c|c|c|c|c|}
\hline & & Fase 1 & Fase 2 & Fase 3 & Fase 4 \\
\hline & Luzes & & Respostas & Reforçadas & \\
\hline \multirow[t]{2}{*}{ Condição 1} & VR & $=$ & $\#$ & $=$ & \# \\
\hline & $\mathrm{AM}$ & $\#$ & $=$ & $\#$ & $=$ \\
\hline Conseqüência para acerto & & Bombom & Bombom & Bombom & Bombom \\
\hline Conseqüência para erro & & Extinção & Som & Extinção & Extinção \\
\hline \multirow[t]{2}{*}{ Condição 2} & VR & $=$ & $\#$ & $=$ & $\#$ \\
\hline & $\mathrm{AM}$ & $\#$ & $=$ & $\#$ & $=$ \\
\hline Conseqüência para acerto & & Bombom & Bombom & Bombom & Bombom \\
\hline Conseqüência para erro & & Extinção & Extinção & Som & Extinção \\
\hline \multirow[t]{2}{*}{ Condição 3} & VR & $=$ & \# & $=$ & \# \\
\hline & $\mathrm{AM}$ & $\#$ & $=$ & $\#$ & $=$ \\
\hline Conseqüência para acerto & & Certo & Certo & Certo & Certo \\
\hline Conseqüência para erro & & Extinção & Errado & Extinção & Extinção \\
\hline \multirow[t]{2}{*}{ Condição 4} & VR & $=$ & $\#$ & $=$ & \# \\
\hline & $\mathrm{AM}$ & $\#$ & $=$ & $\#$ & $=$ \\
\hline Conseqüência para acerto & & Certo & Certo & Certo & Certo \\
\hline Conseqüência para erro & & Extinção & Extinção & Errado & Extinção \\
\hline
\end{tabular}

Nota. VR = Luz vermelha; AM = Luz amarela.

Na Condição 1, Fase 1, a instrução correspondente era apresentada como se segue: $\mathrm{O}$ experimentador apresentava ao participante um arranjo de estímulos, apontava para o cartão modelo e dizia: "Este é o cartão-mãe. Toque com o dedo o cartão-mãe”. Após o participante tocar com o dedo o cartão-mãe, o experimentador apontava para os dois cartões de comparação e dizia: "Este são os cartões-filhos. Toque com o dedo os cartões-filhos". Após o participante tocar com o dedo os cartões-filhos, o experimentador acendia a luz vermelha e dizia: "Quando a mesa ficar vermelha, você deve tocar com o dedo o filho que é igual à mãe. A mesa está vermelha, toque com o dedo o filho que é igual à mãe". Após o participante tocar, o experimentador dizia: "Fazendo isso, você ganha um bombom que eu tiro aqui da minha vasilha e coloco na sua”. Em seguida o experimentador acendia a luz amarela e dizia: "Quando a mesa ficar amarela, você deve tocar com o dedo o filho que é diferente da mãe. A mesa está amarela, toque com o dedo o filho que é diferente da mãe”. Após o participante tocar, o experimentador dizia: "Fazendo isso, você ganha um bombom que eu tiro aqui da minha vasilha e coloco na sua". Esta instrução era apresentada mais uma vez ao participante. Após a apresentação desta instrução, o experimentador informava ao participante que não podia mais conversar com ele durante o jogo.

Na Fase 1 eram reforçadas com bombons as respostas de tocar o estímulo de comparação igual ao modelo quando a luz vermelha estivesse acesa e as respostas de tocar o estímulo de comparação diferente quando a luz amarela estivesse acesa (respostas corretas). As respostas incorretas não eram conseqüenciadas pelo experimentador. Na Fase 2, as contingências programadas eram revertidas, passando-se a reforçar com bombons as respostas de tocar o estímulo de comparação igual ao modelo quando a luz amarela estivesse acesa e as de tocar o diferente quando a luz vermelha estivesse acesa (respostas corretas). As respostas incorretas eram conseqüenciadas com a apresentação do estímulo sonoro. Na Fase 3, as contingências em vigor na Fase 1 eram restabelecidas. Nesta fase, portanto, as respostas corretas produziam bombons e as incorretas não eram conseqüenciadas pelo experimentador. Na Fase 4, as contingências eram novamente revertidas, tal como foi feito na Fase 2. Mas na Fase 4, enquanto a emissão das respostas corretas continuava produzindo bombons, a emissão das 
respostas incorretas não era conseqüenciada pelo experimentador.

A Condição 2 diferia da Condição 1 apenas com relação às conseqüências programadas para as respostas corretas e incorretas nas Fases 2 e 4 . Na Fase 2 da Condição 2, as respostas corretas eram conseqüenciadas com bombons e as respostas incorretas não eram conseqüenciadas pelo experimentador. Na Fase 4 , as respostas corretas eram conseqüenciadas com bombons e as respostas incorretas eram conseqüenciadas com a apresentação do estímulo sonoro.

Na Condição 3, tal como nas Condições 1 e 2, a Fase 1 também era iniciada com a apresentação da instrução correspondente. A instrução correspondente apresentada na Condição 3, no entanto, diferia da apresentada nas Condições 1 e 2, no seguinte aspecto: Na Condição 3, no lugar de dizer "Fazendo isso, você ganha um bombom que eu tiro aqui da minha vasilha e coloco na sua", o experimentador dizia "Quando você fizer isso eu direi certo”. Na Condição 3, as respostas consideradas corretas e incorretas e as conseqüências programadas para todas as fases eram as mesmas da Condição 1, apenas a natureza das conseqüências apresentadas era diferente. $\mathrm{Na}$ Condição 3 , a emissão das respostas corretas era conseqüenciada com a verbalização “Certo”. Já a emissão das respostas incorretas era conseqüenciada com a apresentação do estímulo verbal "Errado" na Fase 2, e não era conseqüenciada pelo experimentador nas Fases 1, 3 e 4.

A Condição 4 diferia da Condição 3 apenas com relação às conseqüências programadas para as respostas corretas e incorretas nas Fases 2 e 4 . Na Fase 2 da Condição 4, as respostas corretas eram conseqüenciadas com "Certo" e as respostas incorretas não eram conseqüenciadas pelo experimentador. $\mathrm{Na}$ Fase 4, as respostas corretas eram conseqüenciadas com "Certo" e as respostas incorretas eram conseqüenciadas com "Errado".

Nas quatro condições, a Fase 1 era encerrada após a apresentação de 30 tentativas e cada uma das Fases 2, 3 e 4 era encerrada após um de dois critérios ser atingido, o que ocorresse primeiro: a) a emissão de 10 respostas corretas consecutivas ou, b) a ocorrência de 30 tentativas. Nas quatro condições, quando uma resposta era reforçada, era reforçada em esquema de reforço contínuo (CRF). Em todas as fases, as luzes eram apresentadas aleatoriamente e quando uma luz estava acesa a outra ficava apagada. Cada participante era exposto a uma única sessão que durava 40 minu- tos, aproximadamente, e era encerrada ao final da Fase 4. Ao término da sessão o experimentador entregava um brinde (brinquedo e/ou guloseima) para a criança.

\section{RESULTADOS}

As Figuras 1, 2, 3 e 4 apresentam a freqüência acumulada de respostas corretas e respostas incorretas emitidas por cada participante durante as quatro fases das Condições 1, 2, 3 e 4.

Na Figura 1, observa-se que todos os participantes (P11, P12, P13 e P14) seguiram a instrução apresentada no início da Fase 1. Na Fase 2, quando as contingências programadas mudaram e a manutenção do comportamento de seguir a instrução passou a produzir o estímulo sonoro, três (P11, P12 e P13) dos quatro participantes deixaram de seguir a instrução e passaram a emitir respostas corretas, de acordo com as novas contingências. Isto é, passaram a ganhar bombons por não seguirem a instrução. Na Fase 3 , quando as contingências em vigor na Fase 1 foram restabelecidas, isto é, quando o não-seguimento da instrução deixou de produzir bombons e a emissão do comportamento que havia sido previamente especificado pela instrução no início da Fase 1 voltou a produzir bombons, os Participantes P11 e P13 mudaram seus desempenhos acompanhando a mudança nas contingências. Já o Participante P12 não mudou; continuou emitindo o mesmo desempenho da Fase 2. Na Fase 4, P11 e P13 mudaram novamente seus desempenhos de acordo com a mudança nas contingências e P12 variou seu desempenho durante toda a fase. O Participante P14 seguiu a instrução apresentada na Fase 1 em todas as fases subseqüentes do experimento.

Na Figura 2, observa-se que todos os participantes da Condição 2 seguiram a instrução apresentada na Fase 1. Na Fase 2, quando as contingências foram alteradas e o seguir a instrução deixou de produzir bombons, três (P21, P23 e P24) dos quatro participantes continuaram seguindo a instrução, mantendo o mesmo desempenho na Fase 3. O Participante P22 foi o único que mudou seu desempenho acompanhando a mudança nas contingências nas Fases 2 e 3. Na Fase 4 quando as respostas incorretas passaram a produzir o estímulo sonoro, todos os participantes mudaram seus desempenhos, passando a responder corretamente, de acordo com as contingências. 

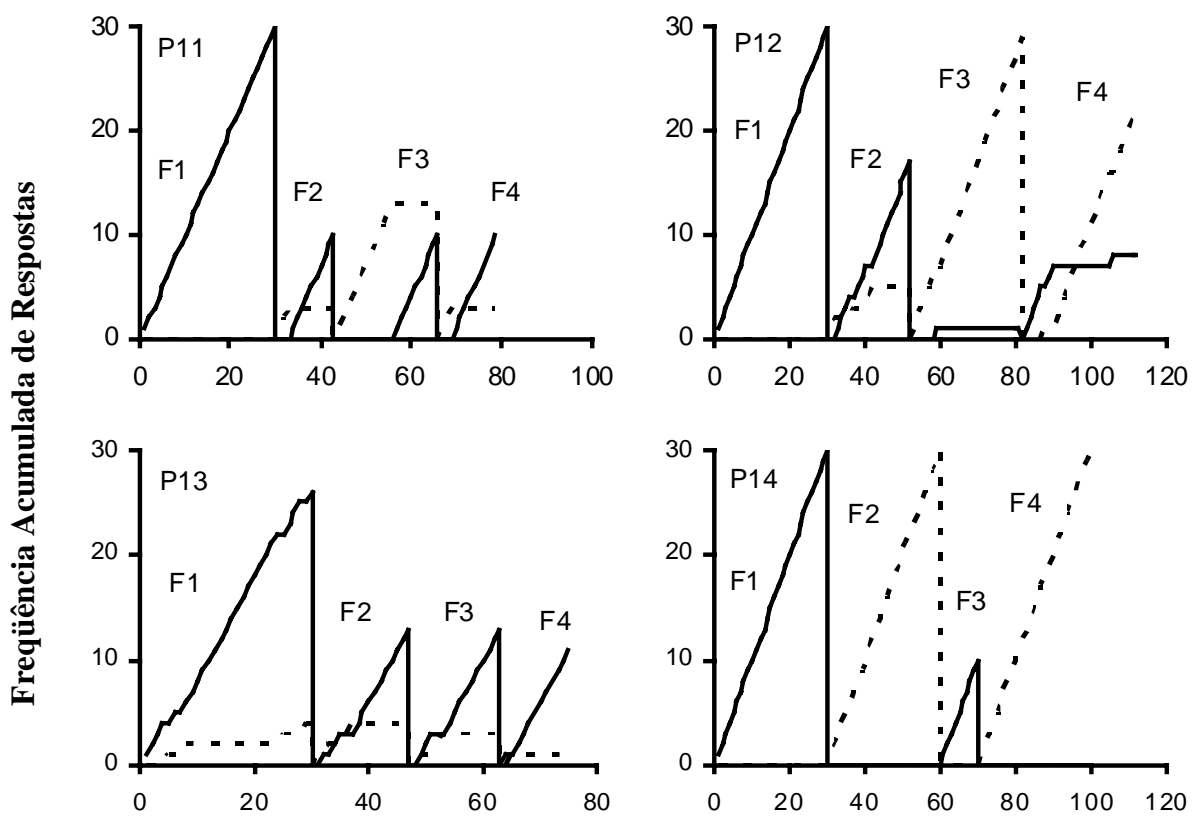

Tentativas

Figura 1. Freqüência acumulada de respostas corretas (linha sólida) e respostas incorretas (linha tracejada), para cada participante (P) da Condição 1, durante cada fase (F) experimental. Quebras na curva acumulada indicam mudanças de fase.

Na Figura 3, observa-se que todos os participantes da Condição 3 iniciaram a Fase 1 emitindo respostas corretas e continuaram seguindo a instrução na maioria das tentativas da fase. Na Fase 2, quando as contingências foram alteradas, isto é, quando o comportamento de seguir a instrução passou a produzir a verbalização "Errado" e o não-seguimento da instrução passou a produzir a verbalização "Certo", todos os participantes iniciaram a fase apresentando o mesmo desempenho da fase anterior. Mas, logo depois, deixaram de seguir a instrução e passaram a responder corretamente sob controle das contingências em vigor na Fase 2. Na Fase 3, quando as contingências foram novamente alteradas, isto é, quando o não-seguimento da instrução deixou de produzir a verbalização "Certo" e a emissão do comportamento que havia sido previamente especificado pela instrução no início da Fase 1 voltou a produzir a verbalização "Certo", os Participantes P32 e P33 continuaram apresentando o mesmo desempenho apresentado na fase anterior e mantiveram este desempenho na Fase 4. O Participante P31 mudou seu desempenho acompanhando a mudança nas contingências nas Fase 3 e 4 e o Participante P34 apresentou um desempenho variável, ora emitindo respostas corretas, ora incorretas, durante as Fases 3 e 4. 

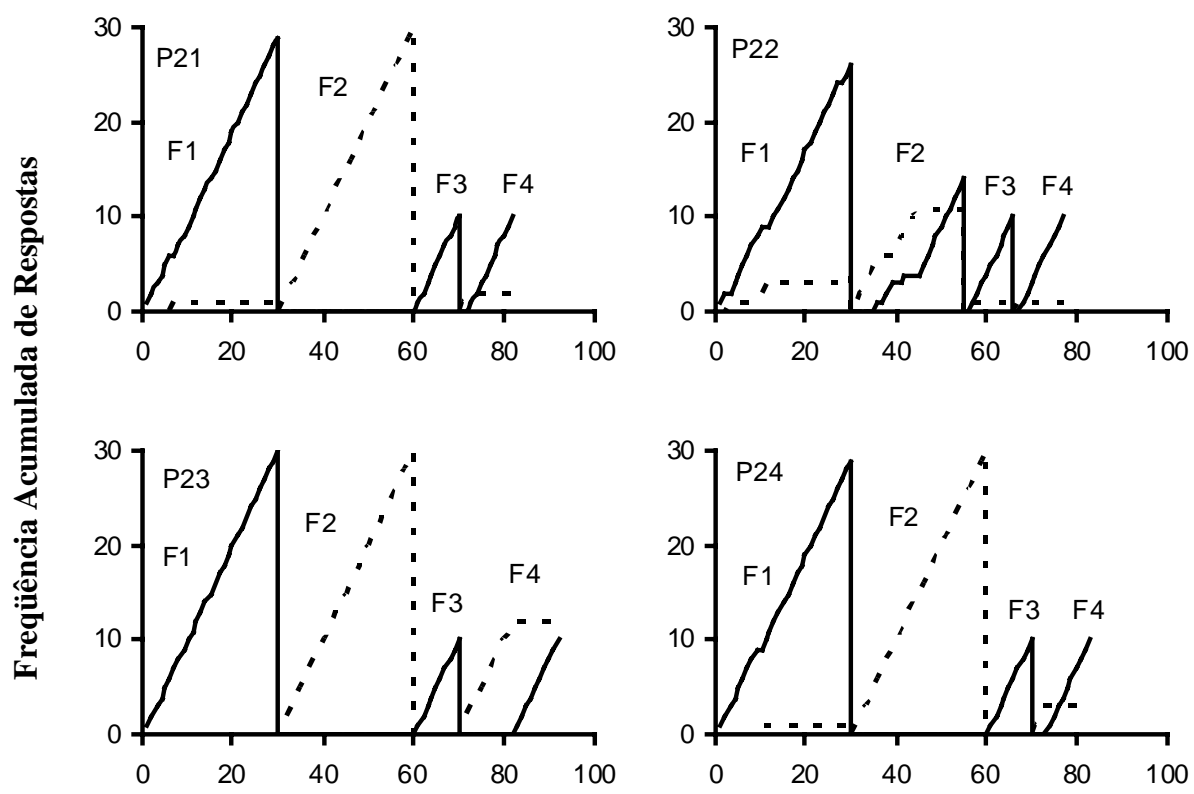

\section{Tentativas}

Figura 2. Freqüência acumulada de respostas corretas (linha sólida) e respostas incorretas (linha tracejada), para cada participante $(P)$ da Condição 2, durante cada fase (F) experimental. Quebras na curva acumulada indicam mudanças de fase.
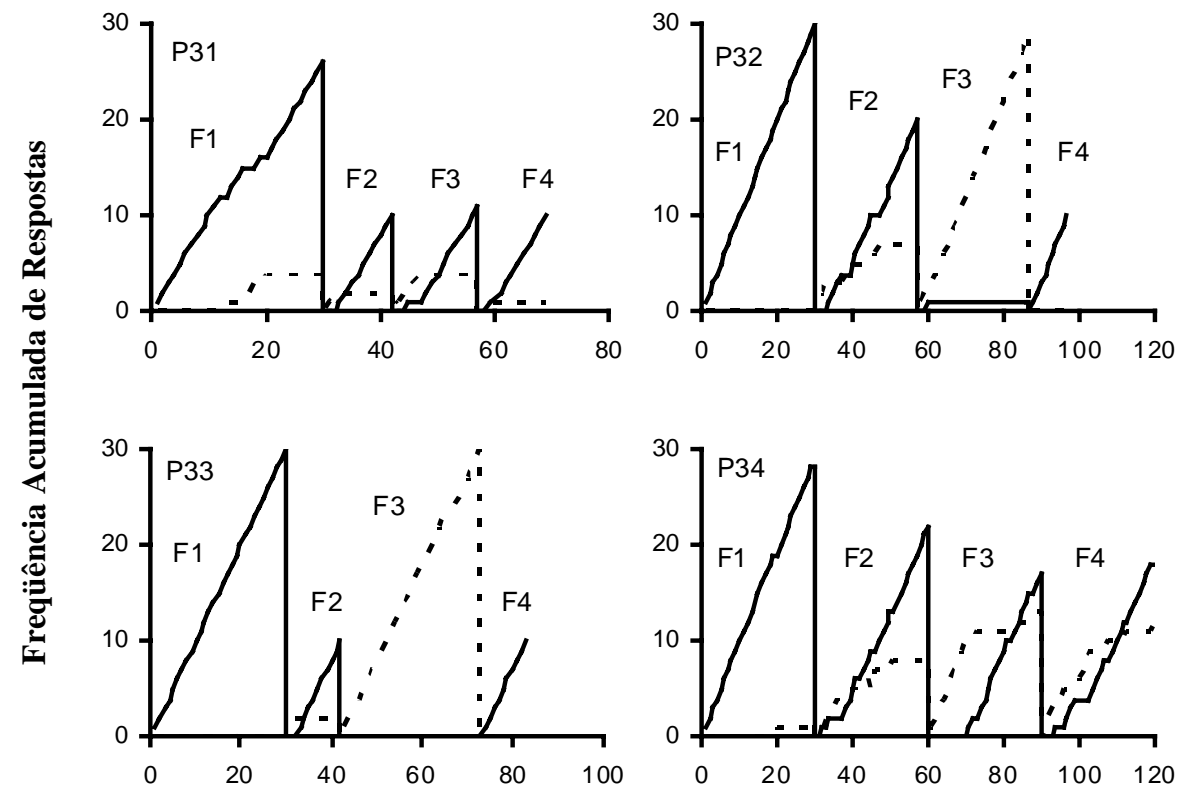

\section{Tentativas}

Figura 3. Freqüência acumulada de respostas corretas (linha sólida) e de respostas incorretas (linha tracejada), para cada participante (P) da Condição 3, durante cada fase (F) experimental. Quebras na curva acumulada indicam mudança de fase. 
Na Figura 4, observa-se que todos os participantes da Condição 4 seguiram a instrução na maioria das tentativas da Fase 1. Na Fase 2, quando a manutenção do seguimento da instrução deixou de produzir o estímulo verbal "Certo", todos os participantes continuaram seguindo a instrução, ou seja, não mudaram seus desempenhos acompanhando a mudança nas contingências. Na Fase 3, com o retorno às contingências em vigor na Fase 1, todos os participantes continuaram seguindo a instrução. Na Fase 4, quando a manutenção do seguimento da instrução passou a produzir a verbalização "Errado" e o não-seguimento passou a produzir a verbalização "Certo", todos os participantes deixaram de seguir a instrução e passaram a apresentar um desempenho sob controle das contingências programadas para a fase.
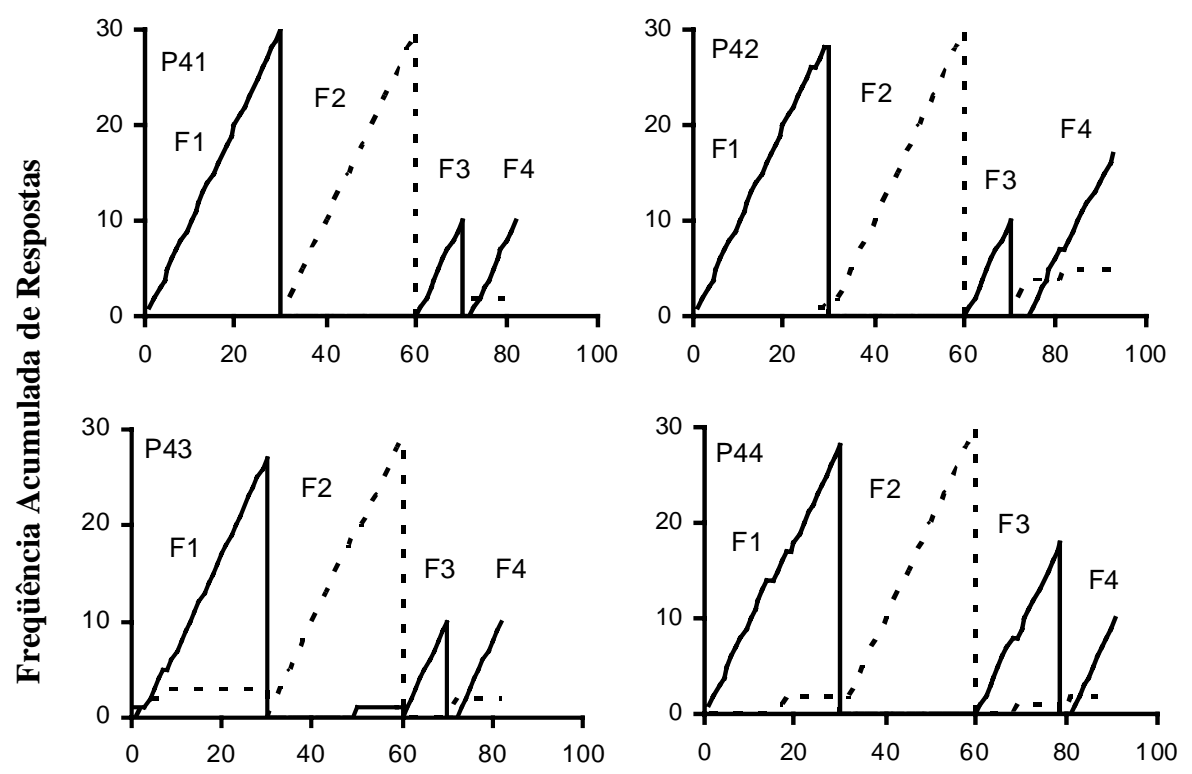

Tentativas

Figura 4. Freqüência acumulada de respostas corretas (linha sólida) e de respostas incorretas (linha tracejada), para cada participante (P) da Condição 4, durante cada fase (F) experimental. Quebras na curva acumulada indicam mudança de fase.

\section{DISCUSSÃO}

O presente estudo, em síntese, teve como objetivo verificar se o seguimento de regras também tende a ser abandonado quando produz outras conseqüências, além de perda de reforçadores. Procurou também verificar se este comportamento tende a ser mantido, após a mudança nas contingências, quando deixa de produzir outras conseqüências reforçadoras, além de pontos e fichas. Os resultados observados na transição da Fase 1 para a Fase 2 das quatro condições mostraram que: a) quando o seguimento da regra passou a produzir um som (caso da Condição 1), três dos quatro participantes deixaram de seguir a regra e quando passou a produzir a palavra "Errado" (caso da Condição 3), todos os quatro participantes também abandonaram o seguimento da regra; e, b) quando o seguimento da regra deixou de produzir bombons (caso da Condição
2), três dos quatro participantes continuaram seguindo a regra e quando deixou de produzir a palavra "Certo" (caso da Condição 4), todos os quatro participantes também continuaram seguindo a regra.

Tais resultados não só apóiam a sugestão de que o seguimento de regras tem maior probabilidade de ser abandonado quando produz conseqüências aversivas do que quando produz outros tipos de conseqüências (Baron \& Galizio, 1983; Chase \& Danforth, 1991; Galizio, 1979; LeFrancois \& cols., 1988; Paracampo \& Albuquerque, 2004; Perone \& cols., 1988), mas também ampliam a generalidade dessa sugestão. Ou seja, no presente estudo, os resultados mostraram que o seguimento de regras tende a ser abandonado, não apenas quando produz a perda de um reforçador, mas também quando produz a apresentação de um estímulo aversivo. Também mostraram que as conse- 
qüências utilizadas aqui exerceram os mesmos efeitos das conseqüências tradicionalmente utilizadas nos estudos do comportamento governado por regras. Tal como a obtenção de pontos ou fichas, os bombons e a palavra "Certo" funcionaram como estímulos reforçadores; e do mesmo modo que a perda de pontos ou fichas, o som e a palavra "Errado" funcionaram como estímulos aversivos.

Além disso, os dados das Fases 2 e 4 das Condições 2 e 4, demonstram, mais claramente, que a manutenção do comportamento de seguir regras depende, em parte, do tipo de conseqüência por ele contatada. Isto pode ser afirmado por três razões principais: a) nestas condições, todos os 7 participantes que seguiram a regra na Fase 2 [quando o seguimento da regra deixou de produzir bombons (caso da Condição 2) ou a palavra "Certo" (caso da Condição 4)], abandonaram o seguimento da regra na Fase 4 [quando este comportamento passou a produzir o som (caso da Condição 2) ou a palavra "Errado" (caso da Condição 4)]; b) não se pode dizer que essas diferenças de resultados tenham ocorrido devido a diferenças individuais entre os participantes, uma vez que as diferenças relativas ao seguimento ou não da regra foram observadas em um mesmo participante; e, c) também não se pode dizer que na Fase 4 o seguimento da regra tenha sido abandonado devido ao tempo de exposição do seguimento da regra à discrepância regra/contingências, porque este comportamento foi reforçado na Fase 3 e, portanto, não foi exposto a esta discrepância nesta fase.

Além das conseqüências programadas para o seguimento da regra, as conseqüências contatadas pelo não-seguimento da regra também contribuíram para determinar os desempenhos dos participantes no presente estudo. Isto fica mais claro quando os resultados das Condições 1 e 3 são analisados. Nas Fases 3 e 4 destas condições, os Participantes P11, P13, P31 e P34 mudaram seus comportamentos acompanhando as mudanças nas contingências, enquanto os Participantes P12, P32 e P33 continuaram emitindo o comportamento apresentado na Fase 2. É possível que essa variabilidade tenha ocorrido porque estes participantes responderam nas Fases 3 e 4 sob controle de suas histórias de exposição às contingências da Fase 2. Ou seja, todos estes participantes deixaram de seguir a regra na Fase 2 porque o seguimento de regra passou a produzir conseqüências aversivas. Mas depois de abandonarem o seguimento da regra, é possível que os desempenhos dos Participantes P11, P13, P31 e P34 tenham ficado sob controle das conseqüências positi- vamente reforçadoras produzidas pelo não-seguimento da regra, enquanto os desempenhos dos Participantes P12, P32 e P33 tenham ficado sob controle das conseqüências reforçadoras de evitar as conseqüências aversivas. Assim, quando as contingências foram alteradas nas Fases 3 e 4, os desempenhos dos Participantes P11, P13, P31 e P34 continuaram sob controle das conseqüências positivamente reforçadoras e mudaram acompanhando as mudanças nas contingências. Já os desempenhos dos Participantes P12, P32 e P33 continuaram sob controle das conseqüências reforçadoras de evitar as conseqüências aversivas e não mudaram acompanhando as mudanças nas contingências.

Essa análise é consistente com a proposição de Paracampo e Albuquerque (2004), que sugere que o contato com as conseqüências diferenciais para o seguimento e para o não seguimento de regra, e não o contato isolado com as conseqüências para o seguimento de regra, é que contribui para determinar a manutenção ou não do seguimento de regra. Ou seja, no presente estudo, o contato do seguimento da regra com as conseqüências aversivas na Fase 2, induziu a ocorrência do não-seguimento da regra, mas a manutenção do comportamento de não seguir a regra foi determinada pelas conseqüências reforçadoras por ele contatadas. No caso das Condições 1 e 3, a história de exposição às contingências da Fase 2, também deve ser considerada na explicação da manutenção dos comportamentos observados nas Fases 3 e 4 . Por essa visão, caso o não-seguimento da regra no presente estudo também tivesse sido punido ou não tivesse sido reforçado, possivelmente, os resultados teriam sido diferentes. Pesquisas futuras poderiam testar estas possibilidades.

Um outro resultado que justifica dizer que apenas em parte o comportamento de seguir regras depende do tipo de conseqüência por ele produzida, foi o obtido no Experimento 2 do estudo de Martinez e Tomayo (2005). Neste experimento, os participantes (estudantes universitários) seguiram uma regra discrepante das contingências mesmo quando o seguimento da regra produzia a palavra "Errado" e o não-seguimento da regra produzia a palavra “Certo”. Este experimento, no entanto, não manipulou as conseqüências programadas para o seguimento de regras, uma vez que elas foram mantidas inalteradas, enquanto que as regras foram manipuladas. Assim, esse resultado possivelmente ocorreu porque os participantes foram expostos a uma história de reforço para o seguimento de regra correspondente às contingências antes de serem expostos à regra discrepante. Uma evidência disso foi 
que na condição em que os participantes só foram expostos à regra discrepante (isto é, em que não foram expostos a essa história de seguimento de regra correspondente), o seguimento da regra discrepante tendeu a ser abandonado. Além disso, neste experimento, as palavras "Certo" e "Errado" eram apresentadas pelo computador, enquanto no presente estudo, eram apresentadas pelo experimentador, que ficava de frente para o participante monitorando o seguimento e o não-seguimento da regra. Esta análise sugere que os participantes do presente estudo foram expostos a uma combinação de condições mais favoráveis ao nãoseguimento de regras do que os participantes do Experimento 2 do estudo de Martinez e Tomayo. Ou seja, sugere que o seguimento de regras é um fenômeno determinado, não exclusivamente por um único fator, mas pela combinação de condições favoráveis e nãofavoráveis a sua ocorrência (L. C. Albuquerque \& cols., 2003).

A história dos participantes de exposição a conseqüências sociais para o seguimento e para o nãoseguimento de regras também deveria ser considerada na análise dos resultados do presente estudo, uma vez que tem sido sugerido que essa história compete com o controle pelas contingências atuais, programadas em situações experimentais (S. C. Hayes \& cols., 1986). É possível admitir que a história pré-experimental de seguimento de regras dos participantes tenha contribuído para que os Participantes P14, P21, P23, P24, $\mathrm{P} 41, \mathrm{P} 42, \mathrm{P} 43$ e P44 tivessem continuado seguindo a regra, quando o comportamento por ela especificado deixou de ser reforçado na Fase 2. Mas a história experimental de reforço para o seguimento de regra, construída na Fase 1, também pode ter contribuído para que o seguimento da regra fosse mantido. Além disso, os dados das Fases 2 e 4 dos Participantes P21, P23, P24, P41, P42, P43 e P44 sugerem que se a história pré-experimental exerce controle, esse controle depende das variáveis atuais, as quais os participantes são expostos, como tem sido sugerido (L. C. Albuquerque \& cols., 2003; V. L. Oliveira \& Albuquerque, 2007; Sidman, 1960).

No caso do Participante P14, pode-se especular que o eventual controle exercido por sua suposta história pré-experimental de reforço para o seguimento de regras tenha prevalecido sobre o controle pelas contingências programadas no experimento. Mas o mesmo não pode ser dito em relação ao desempenho do Participante P22, que deixou de seguir a regra na Fase 2. Uma possível explicação para este resultado é que o comportamento desse participante (P22) não ficou sob controle exclusivo da regra na Fase 1, mas sim sob controle da interação entre a regra e as contingências e isto pode ter favorecido o abandono do comportamento de seguir regra na Fase 2. Outra possibilidade seria dizer que na história pré-experimental desse participante o seu comportamento de não seguir regras foi freqüentemente reforçado e ele respondeu sob controle dessa sua suposta história. Na literatura, alguns autores têm procurado avaliar o controle por histórias pré-experimentais, inferindo os seus efeitos a partir das respostas dos participantes ao questionário construído por Rehfisch (1958). Por exemplo, Pinto, Paracampo e Albuquerque (2006) e Wulfert, Greenway, Farkas, Hayes e Dougher (1994) encontram resultados que indicam que participantes (estudantes universitários) previamente classificados (de acordo com as suas respostas àquele questionário) de inflexíveis são mais prováveis de seguir regras discrepantes das contingências do que participantes classificados de flexíveis. Pesquisas futuras deveriam verificar se isso também ocorreria com crianças.

Em síntese, há evidências experimentais que indicam que a manutenção do comportamento de seguir regras discrepantes das contingências depende, em parte, de se este comportamento: (a) é antecedido ou não por uma história de reforço para o seguimento de regra correspondente às contingências (Martinez \& Tomayo, 2005); (b) é antecedido ou não por uma história de variação comportamental (LeFrancois \& cols., 1988); (c) é antecedido ou não por uma história de reforço contínuo do comportamento alternativo ao especificado pela regra (L. C. Albuquerque \& cols., 2006; V. L. Oliveira \& Albuquerque, 2007); e (d) se este comportamento alternativo foi mantido por um período relativamente prolongado ou não (L. C. Albuquerque, Reis \& Paracampo, no prelo). A manutenção do comportamento de seguir regras também depende, em parte, de: (a) se este comportamento mantém ou não contato com a discrepância entre a regra e as contingências programadas (Galizio, 1979); (b) se mantém contato com essa discrepância por um período prolongado ou não (Bernstein, 1988; Michael \& Bernstein, 1991); (c) se ele é monitorado ou não (Barret \& cols., 1987; Cerutti, 1994); (d) se o nãoseguimento de regra é reforçado ou não (L. C. Albuquerque \& cols., 2004; V. L. Oliveira \& Albuquerque, 2007; Paracampo \& Albuquerque, 2004); e (e) de se a discrepância entre a regra e as conseqüências produzidas pelo seguimento de regra é facilmente discriminável ou não (Cerutti, 1991; Newman \& cols., 1985). O presente estudo mostrou, em um mesmo participante, 
que a manutenção do comportamento de seguir regras também depende, em parte, da aversividade das conseqüências por ele produzidas. Além disso, os dados do presente estudo ampliam o conhecimento acerca das condições sob as quais o seguimento de regras é mais ou é menos provável de ser mantido, na medida em que utilizou conseqüências diferentes das tradicionalmente usadas na literatura.

\section{REFERÊNCIAS}

Albuquerque, L. C. (2001). Definições de regras. Em H. J. Guilhardi, M. B. B. P. Madi, P. P. Queiroz, P. P. \& M. C. Scoz (Orgs.), Sobre comportamento e cognição: Expondo a variabilidade - Vol. 7 (pp.132-140). Santo André: ARBytes.

Albuquerque, L. C. (2005). Regras como instrumento de análise do comportamento. Em L. C. Albuquerque (Org.), Estudos do comportamento (pp.143-176). Belém: Edufpa.

Albuquerque, L. C., de Souza, D. G., Matos, M. A., \& Paracampo, C. C. P. (2003). Análise dos efeitos de histórias experimentais sobre o seguimento subseqüente de regras. Acta Comportamentalia, 11, 87-126.

Albuquerque, L. C., Matos, M. A., de Souza, D. G., \& Paracampo, C. C. P. (2004). Investigação do controle por regras e do controle por histórias de reforço sobre o comportamento humano. Psicologia: Reflexão e Crítica, 17, 395-412.

Albuquerque, L. C., Reis, A. A., \& Paracampo, C. C. P. (2006). Efeitos de uma história de reforço contínuo sobre o seguimento de regra. Acta Comportamentalia, 14, 47-75.

Albuquerque, L. C., \& Silva, F. M. (2006). Efeitos da exposição a mudanças nas contingências sobre o seguir regras. Psicologia: Teoria e Pesquisa, 22, 101-112.

Albuquerque, L. C., Reis, A. A., \& Paracampo, C. C. P. (no prelo). Efeitos de histórias de reforço, curtas e prolongadas, sobre o seguimento de regras. Acta Comportamentalia.

Albuquerque, N. M. A., Paracampo, C. C. P., \& Albuquerque, L. C. (2004). Análise do papel de variáveis sociais e de conseqüências programadas no seguimento de instruções. Psicologia, Reflexão e Crítica, 17, 31-42.

Andronis, P. (1991). Rule-governance: Enough to make a term mean. Em L. J. Hayes \& P.N. Chase (Orgs.), Dialogues on verbal behavior (pp.226-235). Hillsdale, N J: Erlbaum.

Baron, A., \& Galizio, M. (1983). Instructional control of human operant behavior. The Psychological Record, 33, 495-520.

Baron, A., Kaufman, A., \& Stauber, K. (1969). Effects of instructions and reinforcement-feedback on human operant behavior. Journal of the Experimental Analysis of Behavior, 12, 701-712.

Barret, D. H., Deitz, S. M., Gaydos, G. R., \& Quinn P. C. (1987). The effects of programmed contingencies and social conditions on responses stereotipy with human subjects. The Psychological Record, 34, 489-505.

Bernstein, D. J. (1988). Laboratory lore and research practices in the experimental analysis of human behavior: Designing session logistics-how long, how often, how many? The Behavior Analyst, 11, 51-58.
Braga, M. V. N., Albuquerque, L. C., \& Paracampo, C. C. P. (2005). Análise dos efeitos de perguntas e de instruções sobre o comportamento não-verbal. Interação em Psicologia, 9, 77-89.

Catania, A. C. (1998). Learning. New Jersey: Prentice Hall.

Catania, A. C., Matthews, A., \& Shimoff, E. (1990). Properties of rule-governed behaviour and their implications. Em D. E. Blackman \& H. Lejeune (Orgs.), Behaviour analysis in theory and practice: Contributions and controversies (pp.215-230). Brighton, UK: Erlbaum.

Catania, A. C., Shimoff, E., \& Matthews, A. (1989). An experimental analysis of rule-governed behavior. Em S. C. Hayes (Org.), Rule-governed behavior: Cognition, contingencies, and instructional control (pp.119-150). New York: Plenum.

Cerutti, D. T. (1989). Discrimination theory of rule-governed behavior. Journal of the Experimental Analysis of Behavior, 51, 259-276.

Cerutti, D. T. (1991). Discriminative versus reinforcing properties of schedules as determinants of schedule insensitivity in humans. The Psychological Record, 41, 51-67.

Cerutti, D. T. (1994). Compliance with instructions: Effects of randomness in scheduling and monitoring. The Psychological Record, 41, 51-67.

Chase, P. N., \& Danforth, J. S. (1991). The role of rules in concept learning. Em L. J. Hayes \& P. N. Chase (Orgs.), Dialogues on verbal behavior (pp.205-225). Hillsdale, NJ: Erlbaum.

DeGrandpre, R. J., \& Buskist, W. F. (1991). Effects of accuracy of instructions on human behavior: Correspondence with reinforcement contingencies matters. The Psychological Record, 41, 371-384.

Dixon, M. R., \& Hayes, L. J. (1998). Effects of differing instructional histories on the resurgence of rule-following. The Psychological Record, 48, 275-292.

Galízio, M. (1979). Contincency-shaped and rule-governed behavior: Instructional control of human loss avoidance. Journal of Experimental Analysis of Behavior, 31, 53-70.

Hayes, S. C. (1986). The case of the silent dog-verbal reports and the analysis of rules: A review of Ericsson and Simon's "Protocol Analysis: Verbal reports as data.” Journal of Experimental Analysis of Behavior, 45, 351-363.

Hayes, S. C., \& Hayes, L. J. (1989). The verbal action of the listener as a basis for rule-governance. Em S. C. Hayes (Org.), Rule governed behavior: Cognition, contingencies, and instructional control (pp.153-190). New York: Plenum.

Hayes, S. C., Brownstein, A. J., Zettle, R. D., Rosenfarb, I., \& Korn, Z. (1986). Rule governed behavior and sensitivity to changing consequences of responding. Journal of the Experimental Analysis of Behavior, 45, 237-257.

Hayes, L. J., Thompson, S., \& Hayes, S. C. (1989). Stimulus equivalence and rule following. Journal of Experimental Analysis of Behavior, 52, 275-291.

Hayes, S. C., Zettle, R., \& Rosenfarb. I. (1989). Rule-following. Em S. C. Hayes (Org.), Rule governed behavior: Cognition, contingencies, and instructional control (pp.191-220). New York: Plenum.

Joyce, J. H., \& Chase, P. N. (1990). Effects of response variability on the sensivity of rule-governed behavior. Journal of the Experimental Analysis of Behavior, 54, 251-262. 
Kaufman, A., Baron, A., \& Koop, R. E (1966). Some effects of instructions on human operant behavior. Psychonomic Monograph Supplements, 1, 243-250.

LeFrancois, J. R., Chase, P. N., \& Joyce, J. (1988). The effects of variety of instrucions on human fixed-interval perfomance. Journal of the Experimental Analysis of Behavior, 49, 383-393.

Lowe, C. F. (1979). Determinants of human operant behaviour. Em M. D. Zeiler \& P. Harzem (Orgs.), Advances in analysis of behaviour: Vol. 1 Reinforcement and the organization of behaviour (pp.159-192). Chichester, England: Wiley.

Malott, R. W. (1989). The achievement of evasive goals: Control by rules describing contingencies that are not direct acting. Em S. C. Hayes (Org.), Rule governed behavior: Cognition, contingencies, and instructional control (pp.269-322). New York: Plenum.

Martinez, H., \& Tomayo, R. (2005). Interactions of contingencies, instructional accuracy, and instructional history in conditional discrimination. The Psychological Record, 55, 633-646.

Michael, R. L., \& Bernstein, D. J. (1991). Transient effects of acquisition history on generalization in a matching-to-sample task. Journal of the Experimental Analysis of Behavior, 56, 155-166.

Monteles, K. M. C., Paracampo, C. C. P., \& Albuquerque, L. C. (2006). Efeitos de uma história de reforço contínuo e de conseqüências sociais sobre o seguir regras. Psicologia: Reflexão $e$ Crítica, 19, 186-196.

Newman, B., Buffington, D. M., \& Hemmes, N. S. (1995). The effects of schedules of reinforcement on instruction following. The Psychological Record, 45, 463-476.

O’Hora, D., Barnes-Holmes, D., Roche, B., \& Smeets, P. (2004). Derived relational networks and control by novel instructions: A possible model of generative verbal responding. The Psychological Record, 54, 437-460.

Okoughi, H. (1999). Instructions as discriminative stimuli. Journal of the Experimental Analysis of Behavior, 72, 205-214.

Oliveira, V. L., \& Albuquerque, L. C. (2007). Efeitos de histórias experimentais e de esquemas de reforço sobre o seguir regras. Psicologia: Teoria e Pesquisa, 23, 217-228.

Oliveira, C. I., Castro, J. M. O., Simonassi, L. E., \& Vieira, T. M. (2002). Resolução de problema e descrição de contingências: Efeitos da acurácia das instruções em tarefas sucessivas. Acta Comportamentalia, 10, 65-86.

Otto, T. L., Torgrud, L. J., \& Holborn, S. W. (1999). An operant blocking interpretation of instructed insensitivity to schedule contingencies. The Psychological Record, 49, 663-684.

Paracampo, C. C. P. (1991). Alguns efeitos de estímulos antecedentes verbais e reforçamento programado no seguimento de regra. Psicologia: Teoria e Pesquisa, 7, 149-161.

Paracampo, C. C. P., \& Albuquerque, L. C. (2004). Análise do papel das conseqüências programadas no seguimento de regras. Interação em Psicologia, 8, 237-245.

Paracampo, C. C. P., de Souza, D. G., Matos, M. A., \& Albuquerque, L. C. (2001). Efeitos de mudança em contingências de reforço sobre o comportamento verbal e não verbal. Acta Comportamentalia, 9, 31-55.

Perone, M., Galizio, M., \& Baron, A. (1988). The relevance of animal-based principles in the laboratory study of human oper- ant conditioning. Em G. Davey \& C. Cullen (Orgs.), Human operant conditioning and behavior modification (pp. 59-85). New York: Wiley \& Sons.

Pinto, A. R., Paracampo, C. C. P., \& Albuquerque, L. C. (2006). Análise do controle por regras em participantes classificados de flexíveis e de inflexíveis. Acta Comportamentalia, 14, 171194.

Rehfisch, J. M. (1958). A scale for personality rigidity. Journal of Consulting Psychology, 1, 11-15.

Robles, H. S. M., \& Gil, M., S. C. A. (2006). O controle instrucional na brincadeira entre crianças com diferentes repertórios. Psicologia: Reflexão e Crítica, 19, 197-205.

Santos, J. G. W., Paracampo, C. C. P., \& Albuquerque, L. C. (2004). Análise dos efeitos de histórias de variação comportamental sobre o seguimento de regras. Psicologia: Reflexão $e$ Crítica, 17, 413-425.

Schlinger, H. (1993). Separating discriminative and functionaltering effects of verbal stimuli. The Behavior Analyst, 16, 923.

Schlinger, H., \& Blakely, E. (1987). Function-altering effects of contingency-specifying stimuli. The Behavior Analyst, 10, 4145.

Schlinger, H., \& Blakely, E. (1994). A descriptive taxonomy of environmental operations and its implications for behavior analysis. The Behavior Analyst, 17, 43-57.

Schmidt, A., \& de Souza, D. G. (2002). Seguimento de instruções em ambiente natural: $\mathrm{O}$ papel das conseqüências no estabelecimento de uma classe generalizada. Acta Comportamentalia, $10,45-64$.

Shimoff, E., Catania, A. C., \& Matthews, B. A. (1981). Uninstructed human responding: Sensitivity of low-rate performance to schedule contingencies. Journal of the Experimental Analysis of Behavior, 36, 207-220.

Sidman, M. (1960). Tactics of scientific research. Nova York: Basic Books.

Silva, L. S., \& Albuquerque, L. C. (2007). Efeitos de histórias comportamentais sobre o comportamento de seguir regras discrepantes das contingências. Interação em Psicologia, 11, 1125.

Simonassi, L. E., Tourinho, E. Z., \& Silva, A. V. (2001). Comportamento privado: Acessibilidade e relação com comportamento público. Psicologia: Reflexão e Crítica, 14, 133-142.

Skinner, B. F. (1963). Operant behavior. American Psychologist, 18, 503-515.

Skinner, B. F. (1969). Contingencies of reinforcement: A theoretical analysis. New York: Appleton-Century-Crofts.

Skinner, B. F. (1974). About behaviorism. New York: Alfred A. Knopf.

Skinner, B. F. (1989). Recent issues in the analysis of behavior. Columbus: Merrill Publishing Company.

Streifel, S., Wetherby, B., \& Karlan, G. R. (1976). Establishing generalized verb-noun instruction-following skills in retarded children. Journal of Experimental Child Psychology, 22, 247260. 
Torgrud, L. J., \& Holborn, S. W. (1990). The effects of verbal performance descriptions on nonverbal operant responding. Journal of the Experimental Analysis of Behavior, 54, 273-291.

Weiner, H. (1970). Instructional control of human operant responding during extinction following fixed-ratio conditioning. Journal of the Analysis Experimental of Behavior, 13, 391-394.

Wulfert, E., Greenway, D. E., Farkas, P., Hayes, S. C., \& Dougher, M. J. (1994). Correlation between self-reported rigidity and rule-governed insensivity to operant contingencies. Journal of Applied Behavior Analysis, 27, 659-671.
Zettle, R. D., \& Hayes, S. C. (1982). Rule-governed behavior: A potential theoretical framework for cognitive-behavior therapy. Em P. C. Kendall (Org.), Advances in cognitive-behavioral research and therapy (pp. 73-118). New York: Academic Press.

Recebido: 04/05/2007

Última revisão: 15/09/2007 Aceite final: 15/10/2007

\footnotetext{
Notas:

${ }^{1}$ Esse trabalho teve o apoio do CNPq em forma de auxílio à pesquisa e em forma de concessão de bolsa de produtividade em pesquisa aos dois primeiros autores, de iniciação científica aos dois autores subseqüentes e de doutorado ao último autor.

${ }^{2}$ Regras são estímulos antecedentes verbais que descrevem contingências e podem exercer múltiplas funções. Instruções, promessas e ameaças são alguns dos exemplos de regras. O comportamento é controlado por regras quando ele é estabelecido por uma regra e ocorre independentemente de suas conseqüências imediatas. O comportamento é controlado por contingências, quando ele é estabelecido por suas conseqüências imediatas e ocorre independentemente de uma descrição antecedente das próprias contingências. E o comportamento é controlado pela interação entre regra e contingência, quando é estabelecido por uma regra e sua manutenção depende de suas conseqüências imediatas (Albuquerque, 2001, 2005).
}

\section{Sobre os autores:}

\section{Carla Cristina Paiva Paracampo}

Professor Associado 1 da Universidade Federal do Pará. Bolsista de Produtividade em Pesquisa do CNPq. Coordenadora do Programa de Pós-Graduação em Teoria e Pesquisa do Comportamento da Universidade Federal do Pará.

\section{Luiz Carlos de Albuquerque}

Professor Associado da Universidade Federal do Pará. Bolsista de produtividade em pesquisa do CNPq.

\section{Andréa Fonseca Farias}

Aluna do curso de Psicologia da Universidade Federal do Pará. Bolsista de iniciação científica PIBIC/CNPq.

\section{Bruna Nogueira Carvalló}

Aluna do curso de Psicologia da Universidade Federal do Pará. Bolsista de iniciação científica PIBIC/CNPq.

\section{Ana Rachel Pinto}

Psicóloga, Mestre em Teoria e Pesquisa do Comportamento. Bolsista de doutorado do CNPq.

Endereço para correspondência: Carla Cristina Paiva Paracampo. Rua Oliveira Belo, 238/1702 - Umarizal - CEP 66050-380 Belém/PA. Endereço eletrônico: cparacampo@gmail.com. 